\title{
Clinical Decision Support Systems: The Fascination with Closed-Loop Control
}

\author{
R. M. Gardner \\ Emeritus Professor and former Chair, Department of Medical Informatics, University of Utah, \\ Salt Lake City, Utah, USA
}

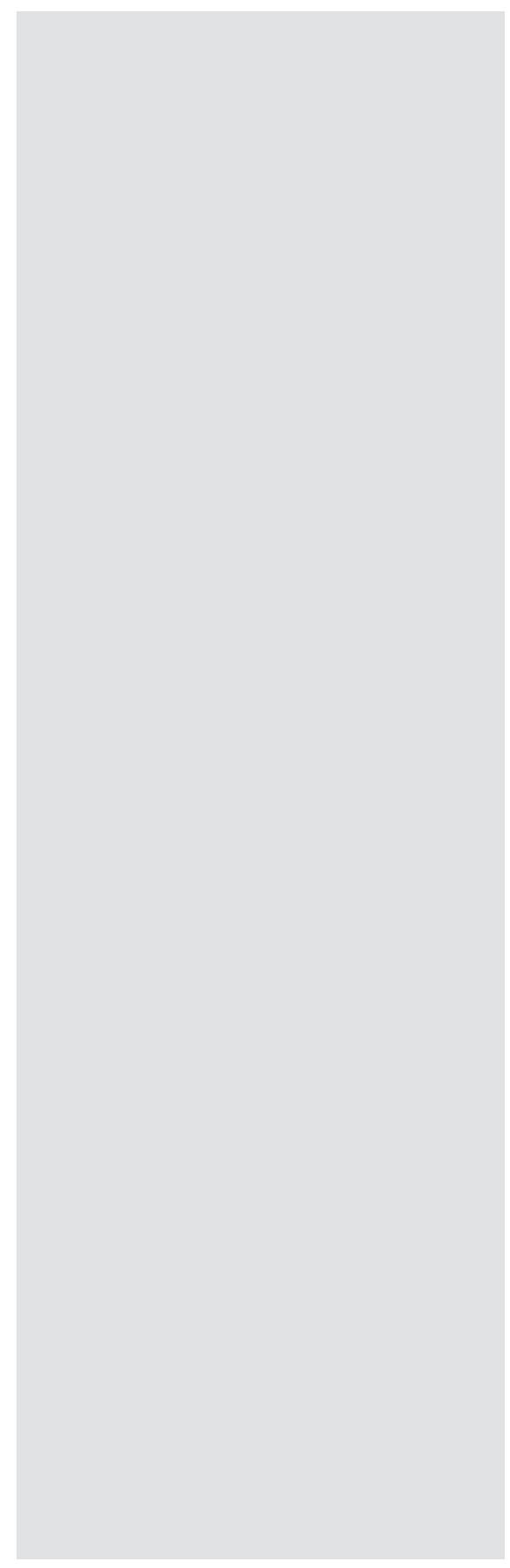

To the engineer a closed-loop control system refers to using a controller with a feedback loop to control states or outputs of a dynamic system. That dynamic system might be the cruise (speed) control on your automobile, maintaining a patient's $\mathrm{paO}_{2}$ with a ventilator, controlling depth of anesthesia with an anesthesia machine, or controlling arterial blood pressure with medications from an IV pump. To a cowboy from the Wild West a closed "loop" might be considered a lariat or lasso rope, used to rope cattle. Similarly, for some in medical informatics, closed-loop control means surrounding a medical problem area to better manage it.

I suggest that, in fact, there is a broad range of Clinical Decision Support (CDS) systems and that closed-loop controllers represent only one specific type. CDS systems include diagnostic systems, intelligent Computerized Physician Order Entry (CPOE), alarming and alerting systems, care advice systems, etc. These CDS systems can be used to help with tasks such as optimizing blood ordering, medication selection and dosing, and using protocols to improve and optimize patient care.

Since the 1950's, when physicians began to understand control system theory, there has been a fascination with having these control systems be closedloop without the need for any human intervention. Bickford at the Mayo Clinic was one of the first to suggest closed-loop anesthesia, using the electroencephalogram (EEG) as the measure [1]. In 1968 Sheppard and his colleagues, at the University of Alabama, showed that the blood pressure of post operative open-heart patient's blood pressures could be controlled with closed-loop strategies [2].

Since those early times, when the use of closed-loop systems was the ultimate decision support strategy, there have been a myriad of other decision support methods developed. However, there still seems to be some magic in being able to "close the loop." Just to give an update on how we are doing some 50 to 60 years later with implementing closed-loop systems, articles in this Yearbook have addressed the topic [3, 4]. Recently, considerable literature on the clinical implementation of closed-loop control has begun to appear. Two examples: a) the accuracy and clinical feasibility closed-loop control of the hypnotic component of anesthesia was recently reported [5]; b) in addition, news reports indicate that closed-loop control of insulin administration for diabetics, the so-called "artificial pancreas," is coming closer to reality [6].

As I was preparing this Keynote manuscript, I began to ask myself why hadn't our group at LDS Hospital in Salt Lake City developed and published ANY work on operational closed-loop control systems. After all, our entire group has hundreds of publications, and most of them are in the field of decision support and Medical Informatics. So, I took the opportunity to refresh my memory on the almost 50 years of Medical Informatics history that I have lived through. It quickly became apparent that as a consequence of working at LDS Hospital with our mentor Homer R. Warner [7] and colleagues T. Allan Pryor, R. Scott Evans, Paul D. Clayton, multiple graduate students and other professional colleagues, we 
have had unique and unusual opportunities in the field of, what I would term, "Operational Clinical Informatics." We were involved in the development and implementation of the HELP Decision Support system and began by using it to computerize Intensive Care Units (ICU) [8]. That experience thrust us into the practice of clinical informatics in medicine. In the process of computerizing this ICU, we attended daily physician/nurse rounds. We watched as data was automatically gathered and stored into computer records and displayed for physicians, nurses and other clinicians to use. Almost immediately it became apparent that the quality of physiological data being collected at the ICU bedsides was highly variable and of intermittent quality. So, to be able to use and display that physiological data collected, we had to fix some of the data collection problems [9]. Soon it became apparent that the physicians and nurses did not understand issues about the need for "timely charting data" and not batch-charting data at hourly intervals or at the end of the shift. Also the frequency of medical data collection and quality improvement methods for data collection had not been widely explored. For example, how often should a blood pressure signal be recorded - every beat, every minute, every 15 minutes or does it depend on other variables? Since we wanted only high-quality data in our computerized record systems, methods for assuring that quality data was collected had to be developed. On subsequent investigation, it was determined that there were few quality standards available for collecting high-quality physiological data. This led us to what is still an active part of my professional life: developing high-quality automated methods for acquiring medical data, developing data collection standards, training and encouraging clinicians to use those strategies to acquire high-quality data for the patient's record.

The establishment of quality data collection methods was crucial in establishing the HELP system as a decision-support tool [10-13]. Soon we were interpreting blood gas results and pulmonary function tests $[14,15]$. It became clear from these interpretation systems that we needed to gather and present data better, as well as integrate data from other data sources. For example, with blood gases, we needed to have data about what nurses and respiratory therapists had been charting on a particular patient. As a consequence, computerized Respiratory Therapy charting was begun [16]. Then as more extensive laboratory data became available, it was possible to consider protocols for improving patient care. These included ventilator weaning protocols $[17,18]$, optimization of blood ordering [19], detecting and preventing adverse drug events $[20,21]$. With coded microbiology data and having medication orders in computerized form, it was then possible to have the computer monitor infectious disease events and make recommendations about the type and dose of antibiotic that should be given. From those activities came the better computerized monitoring of infectious diseases and the antibiotic-assistant [22, 23].

Implementing these decision support systems required very sensitive and careful interaction with the clinicians caring for the patients - physicians, nurses, pharmacists, respiratory therapists, etc. We soon found that social, political and intellectual challenges were very important ingredients to successful implementation of these systems [24, 25]. On many occasions I explained to my colleagues that what we were doing was only about $20 \%$ technology and $80 \%$ sociology. Little did we know we were dealing in the field of human factors research, a field that had its beginnings about the same time that medical informatics began [26]. As the article by Saleem and colleagues in this Yearbook show, human factors and how the system integrates with the care giver are crucial to the successful implementation and value of such systems [27]. A recent editorial by Chaudhry supports the importance of implementation challenges: "The question is not whether computerized decision support systems should or will be integrated into care delivery. Rather, the question of fundamental importance is how can these systems be best used to improve care. If health technology is going to transform healthcare, a deeper understanding of complex dynamics underlying system adoption and use is needed." [28]

Then we were challenged by our medical informatics peers and those who wanted to know a broad answer to the "So what" questions: Were the systems effective? Did users like them? What did they like? What didn't they like and why? Were these systems costeffective and if so, why? As a consequence, we have spent and continue to spend considerable effort to answer these questions. It was rewarding to read, in an Annals of Internal Medicine, an article entitled "Systematic Review: Impact of Health Information Technology on Quality, Efficiency and Cost of Medical Care," published in May 2006 that LDS Hospital was classified as one of the four benchmark hospitals [29].

Perhaps we were distracted by the day-to-day provision of care-using computers. I think not. I think we were motivated by real needs and real opportunities, gained by being immersed in the clinical environment. With that information as background, I will now consider what I think are gaps and opportunities for application of decision support systems and the challenges of implementing better CDS systems, including closed-loop systems.

\section{What Are the Challenges and Opportunities for the Future of Clinical Decision Support, Including Use of Closed-Loop Systems?}

I would classify these challenges into the following five groupings:

1. "It isn't easy!"

2. Clinical Informaticists are needed

3. Major problems with data acquisi- 
tion, integration and coding

4. We need to build and maintain reliable systems

5. There is optimism for the future

\section{1. "It Isn't Easy!"}

I still recall the many times my recently departed colleague Al Pryor said "It isn't easy!" When times got tough during the process of developing and installing the HELP system, he would often exclaim, "It isn't easy!" Indeed, as a review, this 2009 IMIA Yearbook provides plenty of evidence of Dr. Pryor's thoughtful observations. However, one must be careful as they read the literature. Some of the literature would suggest that CDS and closed-loop control systems were items that could be purchased "off the shelf." [30]. However, the people who have built such closed-loop systems that are operational in the clinical care setting have typically taken decades to make those systems work [5].

So why isn't it easy? The human body is made up of very complex physiological and neurological systems with an amazing array of sensors and feedback control systems, which maintain homeostasis. We should not be surprised or discouraged at the complexity of trying to mimic these systems. To implement an operational CDS system we must gather the data needed to make the decision, establish knowledge bases, which provide the basis for making the treatment decisions, and then decide how to execute those decisions. Each of these steps is complex and challenging. For example, controlling glucose levels in outpatients and hospitalized patients, especially ICU patients, has been a clinical focus for over a decade. Recent findings from clinical studies have begun to suggest that glucose levels should not be as tightly controlled as was originally thought [31]. The editorial noted that the reasons for these "jarring results" remain unclear. As a consequence, further research is needed to elucidate the reasons and modify the treatment strategies [31].
Recently the Agency for Health Care Research and Quality (AHRQ) has published two important documents that provide a state of the art survey and a how-to guide for implementing CDS systems [32, 33]. These two documents, which are readily available on the Web, provide an excellent overview and set of references that cover the vastness of the issues that must be addressed when implementing CDS systems. In addition, there are grand challenges and urgings for responsible thought and action when implementing CDS systems $[34,35]$. Other recent literature is also helpful. David Bates and his colleagues at Brigham and Women's Hospital implemented a COPE system and felt that they had made observations that were so important that they called them the "Ten Commandments" [36]. Alan Morris, a colleague at LDS Hospital, has provided a very practical guide to implementing computer protocols [37].

There appear to be differences in how medical devices and computerized decision support strategies are conceived and implemented in the United States and in Europe [38]. European investigators seem to have more liberty to apply experimental devices and clinical software systems than investigators in the United States. In the United States, the Food and Drug Administration regulates clinical software [39]. As a consequence, very careful documentation and validation processes must be carried out to be in compliance with those regulations. It is my opinion that additional medical software regulation will only slow and discourage important and innovative developments.

Finally, since many of the CDS systems that will be installed in the future will likely be commercial systems supplied by vendors, there is still a big unknown about how these vendors will enable the customization that will be needed. It is still not known if those systems will be agile enough to adapt to the CDS needs of each specific hospital. Notably, the results from the four benchmark hospitals reviewed by Chaudhry and associates were based on clinical decision systems that had been "internally developed" [29].

\section{Clinical Informaticists Are Needed}

Safran and colleagues have provided an excellent overview and justification for the need of "Clinical Informaticists" in their section on Research and Education in this Yearbook [40]. The American Medical Informatics Association has recently published the core content and training requirements for what it is hoped will become a new medical specialty: Clinical Informatics [41, 42]. Clearly clinical informaticists will be essential in implementing CDS systems.

Recently, I was with a colleague who was describing his hospital's implementation of a new decision-support module in the clinical setting. As I understood it, the module was a CDS system provided by a commercial vendor, but which needed to be customized to fit the clinical practice of their institution. After a considerable time, this colleague explained to me that he had "parachuted in" a clinical informaticist to help resolve the problem. Once the clinical informaticist was in place, the parties began to better communicate, changes were made in the module, and the system was successfully installed. Using the terminology "parachute in" reminded me of military techniques used in guerilla warfare. Perhaps implementing Computerized Decision Support systems is similar to guerilla warfare. Certainly implementing such systems is challenging and difficult.

\section{Major Problems with Signal Acquisi- tion, Data Integration and Coding}

The process of collecting sufficient, reliable, representative, timely, and appropriate clinical data is still a very difficult task. Real-time data collection is a task that many investigators in the field of Medical Informatics still do not understand. Since our work began in the ICU, we had to develop methods to gather real-time data that was of high- 
quality [9]. Now some 40 years later, we and others are in pursuit of that goal. In the meantime, we have helped develop the "Medical Information Bus" as a data standard [43]. In addition, we have explored gathering data from ventilators and other devices automatically. A recent publication showed that manually charted data had many flaws, despite the fact that respiratory therapists were dedicated and diligent about manually entering their computer charting data [44].

I was surprised and disappointed that the Signal Acquisition, Processing \& Integration section of the Yearbook only dealt with image acquisition [45]. Recent work at Massachusetts Institute of Technology has made a large real-time database publicly available to enable many investigators to try strategies for improving the determination of such simple parameters as heart rate $[46,47]$. This database is a marvelous example of how data-mining techniques can be used [48].

Having systems that are interoperable is a major goal of the field of medical informatics. Yet in most health care systems, integrating patient data from multiple sources is very difficult and next to impossible. For example, having access to data from outpatient record systems may not be available to physicians caring for the patient in an emergency room or a hospital. Rassinoux addresses many of these problems in this Yearbook [49].

Today, much of a patient's medical record is in "free-text" format, either written by hand or entered into the computer. Even with excellent word processor and natural language processors, that data is not available in coded format for decision making.

If the CDS system that one wanted to build were a closed-loop system, the above noted problems of integrated, accurate, and real-time signal availability would be crucial. Very few systems in existence today have the ability to collect such data. Only devices like pacemakers and, hopefully, insulin infusion pumps have that capability. In those cases, only one or two primary physiological signals are used.

\section{We Need to Build and Maintain Reliable Systems}

Lenz has provided a very thoughtful assessment of the state-of-the art and outlined steps that must be taken to provide sustainable healthcare information systems [50]. Taking these steps should prevent the necessity of costly systems replacements every five or ten years. An excellent reference is given in this paper to Sauer who discusses "deciding the future for IS failures; not the choice you might think" [51]. I found these two papers very informative since CDS systems; especially closed-loop control systems require remarkable reliability.

Two recent papers have pointed to what some would consider unusual failure modes: "unintended consequences" and "e-Iatrogenesis" [52, 53]. The issues surrounding the reasons for these failures are complex with details noted in the publications. I suspect that many of these complex issues would have been avoided if well-trained "Clinical Informaticists" been involved in the development and implementation process.

A recent report by the National Academy of Science and a 2009 publication in the Communications of the ACM points to medical software as being particularly undependable [54,55]. The ACM article claims that, although medical software can save lives, it can also kill. Unreliability of medical devices and medical software systems was used as a bad example of dependability. Medical devices such as pacemakers were discussed. However, CDS systems raise many of the same issues. We, in Medical Informatics, must take such challenges of providing highly dependable systems seriously and demonstrate those capabilities in systems we develop.

\section{There Is Optimism for the Future}

Bates and his colleagues have provided us with excitingly optimistic results with their two manuscripts, assessing the effects of Health Information systems technology $[56,57]$. We can still be fascinated and challenged by closedloop control systems, but it is not the be-all and end-all of Medical Informatics. In my opinion, closed-loop control is NOT the ultimate CDS system because remarkable progress has been made with remarkable quality of care improvement by using "open-loop" strategies, which involve having the clinician in the loop [28, 29, 56, and 57].

I am inspired and motivated by the comments made by Goethe almost 200 years ago:

"Knowing is not enough; we must apply.

Willing is not enough; we must do."

$$
\text { Johann Wolfgang von Goethe }
$$

I would add the statement:

Theorizing is not enough: we must find out!

The opportunities and challenges are there for us to develop and clinically apply more and better Computerized Decision Support systems.

\section{References}

1. Bickford RG. Use of frequency discrimination in automatic electroencephalographic control of anesthesia (servo anesthesia). Electroencephalogr Clin Neurophysiol 1951 Feb; 3(1):83-6.

2. Sheppard LC, Kouchoukos NT, Kurtts MA, Kirklin JW. Automated treatment of critically ill patients following operation. Ann Surg 1968 Oct; 168(4):596-604.

3. Gaudinat A. Closing the loops in biomedical informatics: From theory to daily practice. Yearb Med Inform 2009:37-9.

4. Tschopp M, Lovis C. Towards Clinically Available Closed-loop Systems for Mechanical Ventilation in the Intensive Care Unit. Yearb Med Inform 2009:75-80.

5. De Smet T, Struys MM, Neckenbroek MM, Van den Hauwe K, Bonte S, Mortier EP. The accuracy and clinical feasibility of a new Bayesian-based closed-loop system for propofol administration using the bispectral index as a controlled variable. Anesth Analg 2008 Oct;107(4):1200-10.

6. REUTER's news on the WEB - Medtronic highlights new advances in development of ClosedLoop system for diabetes management - 6 Jun 2009.

7. Clayton PD. Presentation of the Morris F. Collen Award to Homer R. Warner, MD, PhD "Why not? Let's do it!'J Am Med Inform Assoc 1995 MarApr; 2(2):137-42.

8. Warner HR, Gardner RM, Toronto AF. Computerbased monitoring of cardiovascular functions in 
postoperative patients. Circulation 1968 Apr;37(4 Suppl):II67-74.

9. Gardner RM, Warner HR, Toronto AF, Gaisford WD. Catheter-flush system for continuous monitoring of central arterial pulse waveform. J of Appl Physiol 1970 Dec;29(6):911-3.

10. Pryor TA, Warner HR, Gardner RM. HELP-A total hospital information system. Proc Annu Symp Comput Appl Med Care (SCAMC) 1980; 4:3-7.

11. Pryor TA, Gardner RM, Clayton PD, Warner HR. The HELP system. Proc Annu Symp Comput Appl Med Care (SCAMC) 1982;6:19-27.

12. Kuperman GJ, Gardner RM, Pryor TA. HELP: A Dynamic Hospital Information System. New York,NY: Springer-Verlag Inc; 1991.

13. Gardner RM, Pryor TA, Warner HR. The HELP System: Functionality Update. Int J Med Inform 1999 Jun;54(3):169-82.

14. Gardner RM, Cannon GH, Morris AH, Olsen KR, Price GA. Computerized blood gas interpretation and reporting system. IEEE Computer 1975; 8(1):39-45.

15. Gardner RM, Clausen JL, Cotton DJ, Crapo RO, Epler GR, Hankinson JL, Johnson RL Jr. Computer guidelines for pulmonary laboratories. Am Rev Respir Dis 1986;134:628-9.

16. Andrews RD, Gardner RM, Metcalf SM, Simmons D. Computer charting: An evaluation of a respiratory care computer system. Respir Care 1985 Aug; 30(8):695-707.

17. Sittig DF, Pace NL, Gardner RM, Beck E, Morris AH. Implementation of a computerized patient advice system using the HELP clinical information system. Comp Biomed Res 1989 Oct;22:474-87.

18. East TD, Bohm SH, Wallace CJ, Clemmer TP, Weaver LK, Orme JF Jr, et al. A successful computerized protocol for clinical management of pressure control inverse ratio ventilation in ARDS patients. Chest 1992 Mar;101(3):697-710.

19. Gardner RM, Laub RM, Golubjatnikov OK, Evans RS, Jacobson JT. Computer Critiqued Blood Ordering Using the HELP System. Comp Biomed Res 1990 Dec;23:514-28.

20. Classen DC, Pestotnik SL, Evans RS, Burke JP. Computerized surveillance of adverse drug events in hospital patients. JAMA 1991 Nov 27;266(20): 2847-51.

21. Classen DC, Pestotnik SL, Evans RS, Lloyd JF, Burke JP.Adverse drug events in hospitalized patients. Excess length of stay, extra cost, and attributable mortality. JAMA 1997 Jan 22-29;277(4):301-6.

22. Evans RS, Gardner RM, Bush AR, Burke JP, Jacobson YA, Larsen RA, et al. Development of a Computerized Infectious Disease Monitor (CIDM) Comput Biomed Res 1985 Apr; 18(2):103-13.

23. Evans RS, Pestotnik SL, Classen DC, Clemmer TP, Weaver LK, Orme JF Jr, et al. A computerassisted management program for antibiotics and other antiinfective agents. N Engl J Med 1998 Jan 22:338(4):232-8.

24. Gardner RM, Lundsgaarde HP. Evaluation of user acceptance of a clinical expert system. JAm Med Inform Assoc 1994 Nov-Dec;1(6):428-38.

25. Friedman CP. A "Fundamental Theorem" of Biomedical Informatics. J Am Med Inform Assoc 2009 Mar;16(2):169-70.
26. Parasuraman R, Wickens CD. Humans: Still vital after all these years of automation. Hum Factors 2008 Jun; 50(3):511-20.

27. Saleem JJ, Russ AL, Sanderson P, Johnson TR, Zhang J, Sittig DF. Current challenges and opportunities for better integration of human factors research with development of clinical information systems. Yearb Med Inform 2009:48-58

28. Chaudhry B. Computerized clinical decision support: will it transform healthcare? Editorial. J Gen Intern Med 2008 Jan; 23 Suppl 1:85-7.

29. Chaudhry B, Wang J, Wu S, Maglione M, Mojica W, Roth E, et al. Systematic review: Impact of health information technology on quality, efficiency and cost of medical care. Ann Intern Med 2006 May 16;144(10):742-52

30. Pauldine R, Beck G, Salinas J, Kaczka DW. Closedloop strategies for patient care systems. J Trauma 2008 Apr; 64(4 Suppl):S289-S294.

31. Inzucchi SE, Siegel MD. Glucose control in the ICU - How tight is too tight? N Engl J Med 2009 Mar 26;360(13):1346-9.

32. Berner ES. Clinical decision support systems: State of the Art. AHRQ Publication No. 09-0069-EF. Rockville, Maryland: Agency for Healthcare Research and Quality. June 2009 - Available on the Web at http://healthit.ahrq.gov/images/ jun09cdsreview/09 0069_ef.html

33. Karsh B-T. Clinical practice improvement and redesign: how change in workflow can be supported by clinical decision support. AHRQ Publication No. 09-0054-EF. Rockville, Maryland: Agency for Healthcare Research and Quality. June 2009 - Available on the Web at http://healthit.ahrq.gov/images/ jun09cdsworkflow/09_0054_ef.html

34. Sittig DF, Wright A, Osheroff JA, Middleton B, Teich JM, Ash JS, et al. Grand challenges in clinical decision support. J Biomed Inform 2008 Apr; 41(2):387-92.

35. Miller RA, Gardner RM, Johnson KB, Hripcsak G. Clinical decision support and electronic prescribing systems: a time for responsible thought and action. J Am Med Inform Assoc 2005 Jul-Aug; 12(4):403-9.

36. Bates DW, Kuperman GJ, Wang S, Gandhi T, Killter A, Volk L, et al. Ten Commandments for effective clinical decision support: making the practice of evidence-based medicine a reality. J Am Med Inform Assoc 2003 Nov-Dec;10(6):523-30.

37. Morris AH, Hirshberg E, Sward KA. Computer protocols: How to implement. Best Pract Res Clin Anaesthesiol 2009 Mar;23(1):51-67.

38. Gardner RM, Beale RJ. Editorial: Pressure to Perform: Is Cardiac Output Estimation from Arterial Waveforms Good Enough for Routine Use? Crit Care Med 2009 Jan;37(1):337-8

39. Miller RA, Gardner RM. Recommendations for Responsible Monitoring and Regulation of Clinical Software. Ann Intern Med 1997 Nov 1;127(9):842-5.

40. Safran C. Informatics Training for Clinicians is More Important than Hardware and Software. Yearb Med Inform 2009:164-5.

41. Gardner RM, Overhage JM, Steen E, Holmes JH, Williamson JJ, Detmer DE; AMIA Board of Directors. Core Content for the Subspecialty of Clinical Informatics. J Am Med Inform Assoc 2009, Mar-
Apr;16(2):153-7.

42. Safran C, Shabot MM, Munger BS, Holmes JH, Steen EB, Lumpkin JR, Detmer DE; AMIA Board of Directors. Program requirements for fellowship education in the subspecialty of clinical informatics. JAm Med Inform Assoc 2009,Mar-Apr;16(2):158-66.

43. Gardner RM, Hawley WH, East TD, Oniki T, Young HFW. Real Time Data Acquisition: Recommendations for the Medical Information Bus (MIB). Int J Clin Monit Comput 1991-1992;8(4):251-8.

44. Vawdrey DK, Gardner RM, Evans RS, Orme JF Jr, Clemmer TP, Greenway L, et al. Assessing Data Quality in Manual Entry of Ventilator Settings. J Am Med Inform Assoc May-June 2007;14(3):295-303.

45. Depeursinge A, Müller H. Sensors, Medical Images and Signal Processing. Yearb Med Inform 2009:81-3.

46. Sun JX, Reisner AT, Saeed M, Heldt T, Mark RG The cardiac output from blood pressure algorithms trial. Crit Care Med 2009 Jan;37(1):72-80.

47. Li Q, Mark RG, Clifford GD. Robust heart rate estimation from multiple asynchronous noisy sources using signal quality indices and a Kalman filter. Physiol Meas 2008 Jan;29(1):15-32.

48. Iavindrasana J, Cohen G, Depeursinge A, Mueller H, Meyer R, Geissbuhler A. Clinical Data Mining: a Review. Yearb Med Inform 2009:121-33.

49. Rassinoux AM. Knowledge Representation and Management: Towards Interoperable Medical Terminologies, Yearb Med Inform 2009:99-102.

50. Lenz R. Information Systems in Healthcare - state and steps towards sustainability - A Position Paper. Yearb Med Inform 2009:63-70.

51. Sauer C. Deciding the future for IS failures: not the choice you might think. In: Currie WL, Galliers B, editors. Rethinking Management Information Systems. Oxford University Press; 1999. p. 279-309.

52. Ash JS, Sittig DF, Dykstra R, Campbell E, Guappone K. The unintended consequences of computerized provider order entry: findings from a mixed methods exploration. Intl J Med Inform 2009 Apr; 78 Suppl 1:S69-76.

53. Sittig DF. A Socio-technical Model of Health Information Technology-related e-Iatrogenesis. AMIA Ann Symp Proc 2008 Nov 6:1209-10.

54. Jackson D, Thomas M, Millett LM; editors: for the Committee on Certifiably Dependable Software Systems; National Academy of Sciences. Software for Dependable Systems: Sufficient Evidence? The National Academies Press; 2007.

55. Jackson D. A direct path to dependable software. Comm ACM 2009 Apr;52(4):78-88.

56. Bates DW. The effects of health information technology on inpatient care. Arch Intern Med 2009 Jan 26:169(2):105-7.

57. Zhou L, Soran CS, Jenter CA, Volk LA, Orav EJ, Bates DW, et al. The Relationship between Electronic Health Record Use and Quality of Care over Time. JAm Med Inform Assoc 2009 Jul-Aug;16(4):457-64.

\section{Correspondence to:}

Dr. Reed M. Gardner

1745 Cornell Circle

Salt Lake City, Utah 84108

USA

E-Mail: Reed.Gardner@hsc.utah.edu 